\title{
El Trabajo de Campo y la noción de ambiente: Una teorización fenomenológica con base en las vivencias de los estudiantes del profesorado de Geografía e Historia de la UPEL-IPC
}

\author{
Fieldwork and the notion of environment: A phenomenological theorization \\ based on experiences of the students of the Geography and History teacher \\ training of the UPEL-IPC
}

\author{
Franklin Núñez Ravelo \\ franklingeove@hotmail.com
}

\author{
María Ugas Pérez \\ mariaeleneaugas_88@hotmail.com
}

Universidad Pedagógica Experimental Libertador-Instituto Pedagógico de Caracas. Departamento de Geografía e Historia. Centro de Investigaciones Estudio del Medio Físico Venezolano

\section{RESUMEN}

El propósito fue develar a partir de las experiencias de los estudiantes en el trabajo de campo, la noción que estos construyen de la categoría ambiente. En tal sentido, se asumió desde la investigación analítica, enmarcada en el paradigma socioconstructivista, bajo un enfoque fenomenológico y con un diseño emergente. Surgen como producto de la sistematización cualitativa: (a) 10 temas esenciales como son: el espacio natural, problemas ambientales, vinculación escuela-comunidad, relevancia de los elementos físico-naturales, zonas de riesgo, dinámica del espacio, problemas locales, impacto antrópico, contaminación y desigualdad social; b) estos fueron clasificados en tres categorías fenomenológicas individuales: fuente de información, componentes físico-natural y componente socio-económico; y (c) finalmente se develó el tema universal, referido a la noción de ambiente. Se asume que el trabajo de campo sustentado en el constructivismo, propicia en los estudiantes la aproximación a una noción compleja de la categoría ambiente, sustentada en comprensión de las interacciones que se producen entre sus elementos bióticos y abióticos.

Palabras clave: trabajo de campo; ambiente; formación docente

\section{ABSTRACT}

The purpose was to reveal, from the students' experiences in the field work, the notion that they build from the environment category. In this sense, it was assumed from the analytical research, framed in the socioconstructivist paradigm, under a phenomenological approach and with an emergent design. They arise as a product of qualitative systematization: (a) 10 essential topics such as: the natural space, environmental problems, school-community linkage, relevance of the physical-natural elements, risk zones, space dynamics, local problems, anthropic impact, pollution and social inequality; b) these were classified into three individual phenomenological categories: source of information, physical-natural components and socio-economic component; and (c) the universal theme was finally revealed, referring to the notion of the environment. It is assumed that field work based on constructivism, encourages students to approach a complex notion of the environmental category, from the understanding of the interactions that occur between their biotic and abiotic elements.

Key words: fieldwork, environment; teacher training 


\section{INTRODUCCIÓN}

En un mundo globalizado, en dónde la tecnología y la información avanzan a ritmos avasallantes, resulta perentorio examinar de manera permanente los programas de formación del profesorado, en lo concerniente a su concepción epistémica-curricular, aunado a la pertinencia de las estrategias didácticas que en su contexto y en atención a sus fines, se ejecutan. Esto, debido a que hoy en día se considera al docente, como un agente promotor de la transformación de la sociedad, por ser el actor fundamental, que conduce el proceso de renovación de los modelos de enseñanza, así como, el mediador por excelencia del proceso de aprendizaje (Fullan, 2002; Aristimuño, 2005; Gutiérrez, 2005; Moliner, Fernández, y Sales, 2005 y Vaillant, 2005).

Siendo así, se asume que es imperante la formación de docentes, cuyo perfil les ubique a la vanguardia del proceso de transformación social, capaz de responder a las demandas en materia educativa, que emplazan a la formación de profesionales integrales, consustanciado con los principios éticos y morales, ambientalmente responsable y humanistas, quienes "Deberán saber y ser capaces de enseñar que el futuro de cada individuo depende del futuro de todos, (...), de ponerse en su lugar y de enseñarles a aprender, de acostumbrarlos a interrogarse y a buscar sus propias respuestas" (Braslavsky, 2002, p. 1)

Sobre este asunto, existen muchos modelos que fungen como lineamientos orientadores para la construcción del perfil del docente del siglo XXI. A tal efecto nos parece interesante la propuesta de Perrenoud (2007), quien sugiere para tal fin, la promoción de 10 competencias básicas: (a) organizar y animar situaciones de aprendizaje, (b) gestionar la progresión de los aprendizajes, (c) diseñar y hacer evolucionar dispositivos de diferenciación, (d) Involucrar a los alumnos en su aprendizaje y su trabajo, (e) trabajar en equipo, (f) Participar en la gestión de la escuela (g) informar e involucrar a los padres de familia, (h) utilizar nuevas tecnologías, (i) afrontar los deberes y los dilemas éticos de la profesión, y (j) organizar su propia formación continua.

Ahora bien, en el ámbito de la formación docente en la especialidad de Geografía e Historia, es reconocido el trabajo de campo como una de las estrategias fundamentales, que favorece no solo la aproximación de los estudiantes a la realidad, sino incluso reafirma su identidad como ciudadanos y como profesionales de la enseñanza (Pulgarín, 1998; Montilla 2005; Rivas y Morales, 2006; Godoy y Sánchez, 2006; Llanos, 2010; Lara, 2011; Kanyampa Nakazwe, 2011; Ramírez, Gouveia y Lozada, 2011; Chew-Hung, 2012, Aichino et. al. 2013 y Núñez Ravelo, 2018).

No obstante, debemos alertar, que dependiendo del enfoque epistémico que sustente la planificación y ejecución de la referida estrategia didáctica, se orientará el proceso de aprendizaje de los estudiantes, y por ende, se potenciará su alcance en relación con las tendencias mundiales en materia de formación docente.

Siendo así, desde el Conductismo, como teoría de la enseñanza que solo aborda el estudio de conductas observables y medibles bajo métodos experimentales-inductistas, por su condición estructuralista1: naturalista ${ }^{2}$,

\footnotetext{
${ }^{1}$ Como modelo epistémico: centra el conocimiento en el estudio de las distintas formas como se presentan los eventos, las distintas relaciones y composiciones que en su conjunto configuran una estructura cualquiera, como capas de la realidad. (Barrera, 2010, p.80)

2 Se hace énfasis en las leyes naturales y se privilegia el instinto. Hace énfasis en la condición natural de los organismos, en interacción bajo la primacía de los instintos, recogido por las leyes que la naturaleza impone. (Barrera, 2010, p.43 y 45)
} 
asociacionista $^{3}$ y anti-constructivista ${ }^{4}$, el aprendizaje es controlado desde el mundo externo al estudiante, este no tiene una participación activa, su rol es pasivo, siendo responsabilidad del docente programar las estrategias de aprendizaje que permitan la transferencia de conocimiento a los aprendices.

Por lo que, entre las principales características vinculadas al proceso de aprendizaje bajo el enfoque conductual, se puede afirmar que: (a) se logra asociando estímulos con respuestas, (b) está en función del entorno, no del individuo que aprende; (c) no es duradero, por lo que requiere ser reforzado, y (d) es memorístico, repetitivo y mecánico, respondiendo a estímulos.

Desde esta perspectiva, el trabajo de campo, tiene como rasgos sine qua non, el constituirse en un recorrido en donde el profesor-experto toma una posición protagónica, proporcionado toda la explicación a los estudiantes, y estos sólo se limitan a registrar la referida información. Cumpliendo con los principios de estímulo-respuesta y reforzamiento, el profesor hará preguntas que los estudiantes deberán responder con apoyo en sus anotaciones. De esta manera, la repetitividad con la que produzcan las respuestas acertadas, reforzará el aprendizaje.

Siguiendo el orden de ideas, pero esta vez desde la corriente Cognitivista, se reconoce a los estudiantes como el centro del proceso educativo, en constante interrelación con los otros elementos del acto didáctico: profesores, contexto, experiencias previas, entre otros.

\footnotetext{
3 un estímulo propioceptivo producido por un reflejo inicial se asocia con el estímulo que produce una segunda respuesta "Para Tolman, la recompensa puede mejorar la ejecución pero no tiene efecto en el aprendizaje, es decir, es un incentivo para que el sujeto produzca las respuestas que ha aprendido." (Álvarez, 2007, p.122)

4 Supone que los sujetos, no tiene control sobre los estímulos que recibe del ambiente (Salas, 2002, p. 13).
}

Destacan entre sus principales características, de acuerdo con lo referido por Vegas (2006), que: (a) refleja el aprendizaje humano a través del tiempo, mediante la práctica, interacción, y haciendo uso de las propias experiencias, basado en un proceso de información, resolución de problemas, y un acercamiento razonable al comportamiento humano; (b) los estudiantes constituyen un ente activo procesador de información, a partir de sus esquemas para aprender $y$ solucionar problemas; (c) los estudiantes organizan representaciones dentro de un sistema cognitivo, las cuales le sirven para sus posteriores interpretaciones de lo real; (d) el aprendizaje es concebido como un proceso activo, interactivo y constante; (e) se centra en las representaciones mentales, atención, percepción, memoria, imaginación, lenguaje y pensamiento; (f) estudia la forma en que las representaciones mentales guían los actos internos y externos de la persona, y (g) analiza el tipo de procesos cognitivos y estructuras mentales que intervienen en la elaboración de representaciones mentales.

De lo anteriormente referido, surge la idea que los trabajos de campo, enmarcados en los preceptos cognitivistas, deben planificarse y ejecutarse de manera estructurada, considerando esquemas existentes y experiencias previas de cada estudiante, por lo que si bien, estos pasan a constituirse en participantes activos, el aprendizaje logrado dependerá de los procesos cognitivos individuales que pongan en marcha para relacionar la información nueva con sus cogniciones. Por lo que el profesor, deberá considerar tal asunto, a fin de promover y guiar a estos en las representaciones cognitivas.

Por otro lado, y en contraposición a las teorías educativas referidas hasta ahora, el Constructivismo, en palabras de Jonassen (1991), supone que el ambiente de 
aprendizaje debe abarcar múltiples perspectivas o interpretaciones de realidad, construcción de conocimiento y actividades basadas en experiencias ricas en contexto.

En efecto, aun cuando el constructivismo suele ser considerado una rama del cognitivismo, ya que ambas teorías asumen el aprendizaje como un proceso mental vinculado al individuo que aprende, difiere de esta, ya que la mayoría de los psicólogos cognitivos consideran que la mente es una herramienta de referencia para el mundo real, mientras que los constructivistas creen que la mente filtra lo que nos llega del mundo, para producir su propia y única realidad (Jonassen, 1991).

En este sentido, el conocimiento es una consecuencia de la interacción entre los procesos cognitivos propios de quien aprende y los elementos que caracterizan o condicionan al objeto aprendido. Siendo así, el estudiante asimila la información y genera estructuras mentales lógicas que le permitan explicar la realidad estudiada.

Desde esta perspectiva, el docente deja a un lado el rol protagónico e incluso su posición como orientador del proceso de aprendizaje, para pasar a ser un promotor del desarrollo y autonomía de quienes construyen sus conocimientos.

Basado en estas premisas, se asume que el trabajo de campo se constituye en un medio de aproximación al aula abierta, que involucra a los estudiantes en el proceso de construcción de sus conocimientos, mediante la reflexión y socialización, que les permitan manifestar de manera lógica las expresiones de sentido, a fin de explicar las condiciones que interactúan en la realidad, $\mathrm{y}$, el conocimiento previo es empleado por el estudiante para el análisis de esta, según las condiciones que presenta el contexto. Por su parte, el docente constructivista, asume una postura de orientador, de motivador del proceso de reflexión y construcción de saberes, fomentando paulatinamente la independencia académico-reflexiva de los estudiantes.

Enmarcados en los preceptos del enfoque constructivista, se planteó como propósito de esta investigación, develar la noción de ambiente que los estudiantes del profesorado de Geografía e Historia de la Universidad Pedagógica Experimental Libertador en su Instituto Pedagógico de Caracas, construyen como parte de sus saberes, a partir de las experiencias vivenciadas durante el trabajo de campo.

\section{MÉTODO}

La investigación es de tipo analítica, enmarcada en el paradigma socioconstructivista, bajo un enfoque fenomenológico y con un diseño emergente. Además, es analítica, ya que se centró en la interpretación de los testimonios que los estudiantes como informantes clave, manifiestan en relación con su experiencia en la participación en trabajos de campo, a fin de develar la noción que estos construyen sobre la categoría ambiente.

Se enmarca dentro del paradigma socioconstructivista, ya que por un lado se concibe la realidad como social y subjetiva, en correspondencia con el mundo interior de los actores sociales y su experiencia en el proceso de construcción de sus saberes; y por otro, el proceso de interpretación al que se hizo referencia en el párrafo anterior, tiene como sustento la sistematización cualitativa, que derivó en una serie de categorías que permiten aproximarnos a la noción de ambiente que construyen los estudiantes.

Es fenomenológica, ya que se asumió como método los momentos husserlianos que constituyen la fenomenología transcendental, entre los que destacan: (a) la etapa previa o clarificación de los presupuestos (Husserl, 
1970), (b) etapa descriptiva (Husserl, 1970), (c) etapa estructural (Husserl, 1900 y 1929), y (d) etapa de socialización de los resultados (Husserl, 1913). Esto a fin de garantizar la sistematicidad en el proceso investigativo, así como la construcción de la aproximación teórica.

Se circunscribió al diseño emergente, toda vez que se asume que la teoría emergió, producto de la sistematización de los testimonios expresados por los informantes.

\section{Informantes}

Se consideraron como informantes a los actores sociales protagonistas del proceso de aprendizaje: Los estudiantes. En este sentido, se recogió e interpretó la experiencia y significado atribuidos por doce estudiantes de la especialidad de Geografía e Historia, que para el momento de la entrevista hayan cursado Geografía Física de Venezuela y con experiencia en participación en trabajos de campo. La razón de este criterio, obedeció a que durante el proceso investigativo, nos dimos cuenta, que este es el único curso de la especialidad de Geografía e Historia en el IPC, en dónde durante la última década los estudiantes, participan en todas sus secciones en un trabajo de campo.

\section{Criterios para la sistematización}

Se respetó plenamente la relación que hace la persona de sus propias experiencias, ya que al tratarse de algo estrictamente vivencial, no habría ninguna razón externa para pensar que ella no vivió, no sintió o no percibió las cosas como dice que lo hizo (Martínez, 1998, p. 168). En tal sentido, en el proceso de sistematización, se procedió a:

1. Oír detalladamente muchos casos similares o análogos.

2. Describir cada uno de ellos.

3. Elaborar una estructura común representativa de esas experiencias: tema central o esencial.

En el cuadro 1, se muestran los pasos y momentos fenomenológicos asumidos durante la investigación: 
Cuadro 1. Pasos y Momentos Fenomenológicos.

\begin{tabular}{l} 
Pasos \\
\hline 1. Lectura de las 12 entrevistas \\
2. Delimitación de los temas centrales en \\
las 12 entrevistas, generándose 10 temas \\
centrales. \\
3. Categorización de los 10 temas centrales, \\
agrupándose en 3 categorías \\
fenomenológicas esenciales/individuales \\
sintetizadas
\end{tabular}

4.- Integración de las 3 categorías esenciales individuales/sintetizadas con base en la consistencia interna $\mathrm{y}$ heterogeneidad externa, en 1categoría fenomenológicas esenciales/universales
Lógica del significado: empleo de la lógica

para llegar a la esencia de los significados contenidos en las 12 entrevistas.

Constitutivo Trascendental: Precisar la

similitud entre los 10 temas centrales que emergieron de la entrevista, surgiendo 3 categorías fenomenológicas esenciales/ individuales sintetizadas.

Lógica del significado: empleo de la lógica para llegar a la esencia de los significados contenidos en las 3 categorías esenciales individuales.

Constitutivo Trascendental:_Precisar la similitud entre las 3 categorías fenomenológicas esenciales sintetizadas, reduciéndose a 1 categorías fenomenológicas esenciales/universales Metafísico de la conciencia: Comprensión de la noción de ambiente vivenciada en el trabajo de campo a partir de la experiencia-vivencia de los estudiantes.

como un elemento articulador, y (c) la vinculación escuela-comunidad, considerando la importancia de la educación como vía para la transformación de la realidad.

En relación con el primero de estos, como ya hemos mencionado, por la naturaleza paradigmática del curso Geografía Física de Venezuela orientada a la lógica positivista, en primera instancia, los estudiantes asumen el propósito ya establecido de estudiar los espacios naturales como fuente de información, de allí que emerjan experiencias, entre las que se incluyen las referidas por los informantes, quienes expresaron:

la parte de las terrazas marina y sobre el levantamiento de la tectónica, nosotros es algo que uno ve aquí en aula pero bueno si más o menos seria reflexión ante los problemas ambientales, 
eso, pero cuando llegas al lugar y ves la realidad que notas que puedes asimilar mejor la información. (Informante 1)

al momento de ir al trabajo de campo, a la ensenada de las Maritas, cuando estábamos en Mochima tu veías la forma tu decías a ok, aquí esta otro, si, si, es lo que tú te imaginabas entonces yo creo que la importancia es que tú lo vivas, lo vea porque una cosa es que tú, porque yo me puedo imaginar, un circulo y con una abertura pequeñita, y al verlo es como cambio tu imaginación con respecto a la forma. (Informante 2)

la Cueva del Guácharo que para mí fue importante el tema que a mí me tocaba y volverme como loca a buscar las estalactitas, las estalagmitas, que si las de barrera, para mí fue muy provechoso. (Informante 3 )

me ayudó a conocer un territorio más de mi país, importante para mí, lo que he conocido durante el 5 día en el trabajo de campo, aparte de eso me dio un conocimiento, si me dejo un conocimiento significativo. (Informante 4)

Esta aproximación a los elementos abióticos, resulta de gran importancia para la formación de los profesionales de la enseñanza de la Geografía, ya que les permite reconocer de primera mano los elementos que caracterizan el paisaje como unidad de estudio, no obstante, con miras a priorizar su comprensión totalística, se hace necesario integrar como elemento para el análisis, los factores que se vinculan la acción antrópica, desde una reflexión ante los problemas sociales, al respecto el Informante 5, nos comentó a modo de ejemplo que: ver a los niños que no están en la escuela a esa edad, me entristeció muchísimo, porque son muchos los niños que no están en la escuela por estar trabajando en la explotación de chipichipi, entonces eso me hace comparar sobre los chicos que están aquí en Caracas estudiando y no lo aprovechan y se quejan por todo, yo les digo ahora: mira tú estás estudiando pero ellos a tu edad o menos están trabajando.

Como se devela, el trabajo de campo aun cuando en esencia está planificado atendiendo la naturaleza físico-geográfica del curso, en su ejecución, proporciona a los estudiantes-docentes información sobre las condiciones socio-económicas, las cuales durante el proceso de establecimiento de las interrelaciones para la construcción de los saberes, permiten un elevado nivel de comprensión integral de la dinámica del espacio en estudio.

De allí que desde la dimensión pedagógica, la información compilada suela ser asumida a fin de generar propuestas que permitan mitigar los impactos, considerando una franca vinculación entre la escuela y la comunidad, tal como lo refiere el Informante 6, al afirmar que:

yo creo que desde el punto de vista educativo si se puede ir mucho más allá, yo creo que todo está en poder hacerle llegar el mensaje a los estudiantes, bueno porque si uno le hace llegar el mensaje de cómo podría afectarle la pesca indiscriminada de un ser vivo, como de los peces o la extracción indiscriminada de esas Pepitonas y cómo podría afectar la diversidad, partiendo de estudios más profundos, como podría afectar y se les tiene que hace llegar en los salones de clase, en socializaciones a las comunidades, para que la gente se dé cuenta. 
Siendo así, consideramos que el trabajo de campo, como estrategia pedagógica, alcanza la condición holística, ya que independientemente de la naturaleza de la asignatura para el cual fue planificado, por un lado permite a los estudiantes obtener información sobre las circunstancias espaciales, de modo totalístico, lo que por otro lado, como estaremos profundizando más adelante, los aproxima al conocer de manera vivencial las interrelaciones de los elementos abióticos y bióticos, y a partir de estas, generar propuestas en donde se involucre a la escuela y la comunidad en intervenciones que favorezcan la mitigación de los escenarios adversos que caracterizan a los fenómenos estudiados.

\section{El componente físico-natural del ambiente}

En el contexto de las geociencias, el trabajo de campo generalmente tendrá como propósito identificar, analizar y caracterizar los agentes y procesos que interactúan en la configuración de las condiciones del paisaje

En este sentido, emergieron como producto de la interpretación de las experiencias de los estudiantes en el trabajo de campo, dos temas esenciales: (a) el análisis de los elementos físico-naturales, y (b) el reconocimiento de las zonas de riesgo.

Desde esta perspectiva, el análisis de los elementos físicos-naturales, emerge en un papel preponderante a fin de reconocer las condiciones que presentan los elementos abióticos.

De allí que partiendo de las vivencias en el trabajo de campo, los informantes expresen que:

Los aportes fluviales de los ríos y toda esa geoformas que actualmente se evidencian, como por ejemplo el día que visitamos lo que es la esa llanura de la ciudad de Barcelona, que está en el delta del río Neverí, como se correspondía, pero también hay una diferencia entre ese espacio con respecto la desembocadura del rio Manzanares, que tiene vamos a decirlo así, una desembocadura continua a lo que es la fosa del Cariaco y entonces su aporte de sedimentos se pierde en la fosa. (Informante 2)

explicarle cual es la importancia también que tiene, que brinda estudiar el terreno, estudiar el ambiente. (Informante 4)

Lo que puede haber en la Laguna de Tacarigua, como el Mangle rojo, las dunas, el Castillo de Araya, la Cueva del Guácharo (Informante 5)

las cosas por acantilados, los valles inundados, las costas de bahías que existían, nos dicen que un acantilado o montaña que cae al mar y es erosionada por el efecto del oleaje, pero nosotros como imaginarse, yo por ejemplo soy del Táchira, no hay playas, ósea nunca había visto un acantilado como se observa en el trabajo de campo, de verdad uno ve cuando el oleaje hace su efecto sobre el acantilado (Informante 6)

llegamos a Tacarigua en la primera parada, vemos la zona del cordón litoral, la propia laguna, los sedimentos en suspensión $y$ en transporte de deriva, discutimos sobre los factores para la formación de ese cordón y si todo coincide. (Informante 8)

allí es donde tú ves que son las facetas triangulares, las montañas, los conos de deyección, este, en donde está la plataforma continental, cuando fuimos al sector de Cariaco, ves como las lagunas están secas porque no hay precipitaciones en el lugar que este osea tantas cosas que hay para desglosar, a bueno este es el cono de deyección por esto es que se forma como un cono, como un ¿Cómo es que 
se llama? Como un abanico aluvial de puros sedimentos que se transportan hacia el mar para mí fue bastante significativo porque yo nunca había visto una playa, un mar que fuesen los primeros cien metros de aguas marrones, y entonces yo incluso en $\mathrm{mi}$ vida sabía que era eso, ahora entiendo que son sedimentos que son aportados por los ríos y por la red hidrográfica que hay en las montañas. (Informante 12)

Destacan de las expresiones anteriores, los agentes (subrayados en este párrafo) y procesos naturales tales como: (a) el transporte de sedimento por parte de los ríos y la consecuente formación de depósitos sobre los cuales se asientan los centros poblados (Barcelona) o la perdida de este material en el fondo de fosas marinas (Fosa de Cariaco), (b) el retroceso de los acantilados por la acción erosiva del oleaje, (c) la formación de barreras arenosas frente a la costa como consecuencia de la distribución del aporte fluvial y su redistribución asociada a la deriva litoral, (d) la disminución del espejo de agua en los cuerpos lacustrinos correspondiéndose con las pocas precipitaciones y alta evaporación registradas, entre otros eventos geomorfológicos que caracterizan la dinámica natural del paisaje.

Ahora bien, cuando a las referidas condiciones naturales se le suman las intervenciones antrópicas no planificadas, se puede estar propiciando algunas zonas de amenazas potenciales, las cuales en opinión de los informantes, obedecen a que:

en la parada el cono de deyección, donde está, el ahí mismo esta una casa, ósea cómo es posible que exista una vivienda si sabemos que las precipitaciones lo puede afectar a esa familia ponerla en riesgo y así hay muchos otros casos que es el acción antrópica la que no está en la tónica con lo que es el espacio físico. (Informante 6)

en esa parada al igual que las otras 2, hay problemas ambientales, la producción de desastre, como lo he mencionado anteriormente, pero históricamente ha sido un problema no consideramos, digamos la población ha tenido que ir retrocediendo por las acciones del oleaje, el Castillo ha ido digamos socavando, digamos que su superficie ha venido abajo, debido a ese oleaje, recorrimos unos metro más y encontramos con casas que de igual forma han tenido que ir retrocediendo, entonces si estamos hablando de un Castillo construido siglos atrás, de esas casa más contemporánea y se siguen retrocediendo, sencillamente no tómanos en cuenta la producción de desastre, que son fenómenos que igual forma van a pasar y cuando intervengamos pues igual, se vuelven en desastre, las paradas que le mencionaba de las rocas embutidas, los conglomerados, es por el hecho, digamos es una pequeña escala, vimos las acciones de los fenómenos que se presentaron en Vargas y a pesar de ello, ni irnos tan lejos, años atrás de Vargas, vimos cómo se presenciaba que es una zona que presenta deslizamiento, en el caso de campo, vimos unas casas al lado de una zona torrencial, entonces ambientalmente no respetamos el espacio nuestro territorio, lo intervenimos y salimos afectados por ignorar su dinámica. (Informante 7)

Se acuerda en el cono de deyección, allí el hombre partió completamente una montaña y construyo en medio, $y$ eso es una formación que uno lo ve a conveniencia de uno, pero allí el material se está conglomerando, entonces eso no debería hacerse, pero hay muchos lugares, por ejemplo en la 
laguna, allí hay viviendas que no deberían estar tan cerca por que llega una cantidad de sedimentos bajando hacia el mar y se arrastra esas viviendas. (Informante 8)

Como se aprecia, el trabajo de campo permite a los estudiantes identificar zonas de riesgos socionaturales, a partir del reconocimiento de los procesos físicos que caracterizan el paisaje, aunado a las condiciones del uso del espacio.

Esta visión, les permite reconocer la diferencia entre fenómenos naturales $\mathrm{y}$ desastres, entendiendo para el primer caso, aquellos que son propio de la dinámica del planeta, pero que se transforma en lo segundo cuando los seres humanos, en el uso del espacio, desconociendo o ignorando los procesos asociados al primero, nos hacemos vulnerables y terminamos afectados por las manifestaciones naturales.

De allí que "el estudio de desastres entonces tiene la finalidad de concientizar a la población sobre su situación de vulnerabilidad y otorgarle los conocimientos necesarios para poder alcanzar condiciones de seguridad" (Romero y Maskrey, 1993, p. 10)

Aunado a lo anterior, debemos destacar que el abordaje de los aspectos físicogeográficos del territorio nacional, permite a los estudiantes percibir situaciones asociadas al deterioro ambiental, entre las que nuestros informantes, destacan al referir que:

El ser humano reutiliza su ambiente y esa dinámica como hace, como se, cómo se va expandiendo de repente en un área geográfica o como también va deteriorando (Informante 5)

I: (...) lo descuidadas que pudieran estar algunos sitios por el uso

E: ¿Cómo cuáles?

I: La Boca, que pasamos por ahí. La gente todavía no tiene conciencia de lo importante que nos esos lugares. Igual que cuando estábamos en el Castillo, que en las Dunas la gente lanzaba desperdicios y no ven lo importante que es eso para nuestra geografía. (Informante 11)

Desde estos testimonios, se desprende la idea referida a que el trabajo de campo, fomenta en los estudiantes una comprensión crítica de los impactos que las acciones antrópicas generan en el medio físico-natural, motivando la reflexión sobre la necesidad de revalorizar la importancia de estos últimos, desde una visión integradora del ambiente, centrada en un proceso de concienciación que sensibilice al ser humano sobre la importancia del uso responsable de los recursos.

\section{El componente socio-económico del ambiente}

Otro conjunto de consideraciones a las que permite aproximarse el trabajo de campo en Geografía, a fines de configurar la noción de la categoría ambiente, está referido al componente socio-económico, desde dónde emergen como temas esenciales: (a) la dinámica del espacio, (b) comprensión de los problemas locales, (c) el impacto de las actividades antrópicas, (d) la contaminación y (e) desigualdad social, como principales situaciones problemáticas.

En lo concerniente al primero de los asuntos mencionados, es necesario que destaquemos lo referido por los informantes, quienes nos comentan que:

fuimos al Parque Pinar y ya uno lo conoce pero si fue importante porque uno lo ve de otras perspectivas, tu ibas al parque e ibas a ver a los animales y por encimita la vegetación pero cuando nosotros fuimos comenzamos a detallar de cómo fue evolucionando el parque y como la población, la parte urbana se fue como metiendo y 
fluyendo en ese parque y vemos que el parque es un pedacito nada más y no debería porque mucha la población ocupo ese espacio que era el parque. (Informante 2)

en esa caminata identificamos varios
elementos dentro de cada parroquia,
identificamos cual es el objeto
dinamizante de cada parroquia, y fue
muy (...) chévere porque hay sectores
que uno no conoce, que permitió ver
como las actividades económica que
influyen para que la población se
dirija hacia ella. (Informante 9 )

En ambos casos, se puede evidenciar que los informantes hacen referencia a como a partir de la interpretación de elementos del paisaje, logran comprender como los seres humanos hemos ocupado a través del tiempo los espacios, expandiendo la influencia urbana sobre las áreas naturales.

En todo caso, este tipo de expansión, por no ser controladas ni planificadas, suelen generar impactos ambientales negativos, por lo que resulta conveniente considerar sus huellas ecológicas, ya que este "análisis refleja los dos aspectos más importantes del reto de la sostenibilidad: el consumo de recursos y el crecimiento de la población" (Universidad Rafael Landívar, 2004. p.259) y cuyo ejemplos de aplicación en el ámbito educativo se ha venido desarrollando en países como "Cánada, para la concienciación y sensibilización frente a la problemática ante todo entre los jóvenes, y con la posibilidad de calcular huellas personales, en hogar o por comunidades con determinados contextos socioeconómicos" (Universidad Rafael Landívar, 2004. p. 260).

Dentro de los principales impactos asociados a las actividades antrópicas, podemos destacar, lo referido por el Informante 5, quien nos comentó:

cual sería el impacto que hace el hombre dentro de ese mismo entorno y el trabajo de campo te brinda eso (...) nosotros de repente podamos hacer como seres humanos, al momento, de cierta forma perturbable con nuestras actividades, eso es lo que también nos brinda el trabajo de campo (...) muchísimos cambios también naturales por lo menos en los dos que estuvimos en Higuerote $y$ hacia el Lago de Valencia vimos también como es el impacto ambiental que hace el hombre en el Lago (...) y lo que ves en campo otra de las cosas es como el hombre va ganando terreno para su propio beneficio, como en el caso del Morro de Barcelona, que se crean esas obras de ingeniería que vimos, para acelerar la colmatación del lugar y la formación del tómbolo para su propio beneficio turístico no sabemos si eso está bien evaluado digo los impactos ambientales (...)es evidenciar la relación hombreambiente, vemos muchos impactos que son generados por el hombre, para mí lo más significativo es que nosotros como docentes hemos vivenciado esos fenómenos naturales como antrópicos y ahora podemos hablar a nuestros estudiantes desde la experiencia, sobre la importancia de usar con racionalidad los recursos naturales, mayormente en mi caso en particular, lo que más me impacto fue ver que en uno de los lugares visitados, pudimos observar que no existe ninguna escuela, y ver como una familia completa, desde el niño hasta el más abuelito, se dedican a la actividad de explotación.

Lo anterior, coincide con lo referido por los informantes 3, 6 y 8, quienes nos comentan que:

llevarlos para que ellos visualicen lo importante que es eso y cómo le podemos sacar todo el provecho y todavía no entendemos eso, nos falta esa cultura de cuidar lo nuestro de 
evitar los impactos al ambiente. (Informante 3)

en el ambiente como tal de campo, nosotros pudimos apreciar diversidades de factores predominantes y si pudimos ver la acción del hombre como perjudica la naturaleza en las regiones que pudimos ver que a veces pasan muchas tragedias que no son naturales, que los fenómenos son naturales pero las tragedias suceden por nosotros como seres humano y como afectamos la dinámica de ese espacio (...) en la fecha de Chacopata, la colecta de materiales que no lo hacen de manera correcta, que también afectan la naturaleza, entonces es importante. (Informante 6)

el ser humano el que coloca todas sus cosas antrópicas allí, en donde están los cauces correspondientes a los depósitos de sedimentos como tal, e incluso la guía dice que incluso las personas que colocaron sus viviendas lejos del rio y jamás pensaron que por donde construyeron pasaron cauces de sedimentos (...) que pasa, lo mismo que paso en Vargas, construimos en zonas que no deberíamos construir eso es antrópico, entonces cuando pasan esos fenómenos geográficos, decimos que es una tragedia, y no es una tragedia profesor, porque eso pasa el hombre interviene un lugar que no debe, no es una tragedia, es un proceso normal que está pasando la geografía en un momento y es normal, la tragedia la inventa el hombre por no tener la conciencia suficiente de construir en zonas en donde no debería. (Informante 8)

Aunado a lo anterior, emergen como uno de los principales problemas geográficosambientales, la contaminación, caracterizados con base en los siguientes eventos demostrativos, según los significados otorgados por los informantes, quienes coinciden en afirmar que:

el primer factor negativo que vi en todos lados, fueron los desechos sólidos, creo que en la cueva del Guácharo fue el único lugar que no, pero en todos los demás había basura por todos lados, parece que la gente no puede ver un barranco por así decirlo porque lo usa para colocar basura. (Informante 2)

lo que todos concordamos que hay mucha contaminación y que no debería pues, se supone que si esos lugares son de gran importancia, para nosotros están demasiado contaminados (...) la Península de Araya veíamos las cárcavas en forma de embudo, eso ve que la población la utilizaba como un botadero de pura basura, eso incluso, lo que no solo afecta el paisaje, sino que además puede enfermar a las personas por eso está cerca de las casas, tapando esa parte. (Informante 3)

una de las cosas que más vimos en campo fue la abundante cantidad de desechos sólidos que perjudica ese espacio y es importante observar que bueno esta predominando que la mayoría de las parada nosotros veíamos desechos sólidos y veíamos como afectan. (Informante 6)

desde las primeras paradas noté desperdicios, en la vía, y en los lugares visitados. (Informante 10)

La gente todavía no tiene conciencia de lo importante que nos esos lugares. Igual que cuando estábamos en el Castillo, que en las Dunas la gente lanzaba desperdicios y no ven lo importante que es eso para nuestra geografía. (Informante 11)

Generalmente las condiciones de contaminación percibidas por los estudiantes, están asociadas al inadecuado manejo de los desechos sólidos así como vertederos de 
sustancias residuales provenientes de la industria, entre otros elementos que mostramos la figura 1, lo cual constituye un grave problema ya que no solo afecta las condiciones estéticas del paisaje, sino que además genera mal olor, desestabiliza el equilibrio ecológico e incluso propicia "problemas de salud pública como la reproducción de ratas, moscas y otros transmisores de enfermedades, así como la contaminación del aire y del agua" (Bustos, 2009, p. 123).
Aunado a lo anterior, debemos destacar lo referido por Azqueta (2002), quien advierte que:

tampoco se puede perder la problemática que genera la producción de residuos en sí misma, con independencia de su carácter tóxico o peligroso. La creciente generación de basura, en efecto, supone la necesidad de un tratamiento adecuado de las mismas que requiere de unos recursos, terrenos, energías (...) muchos de estos residuos depositados en vertederos (...) terminan contaminando el aire, el suelo y los acuíferos por lixiviación" (p. 12).

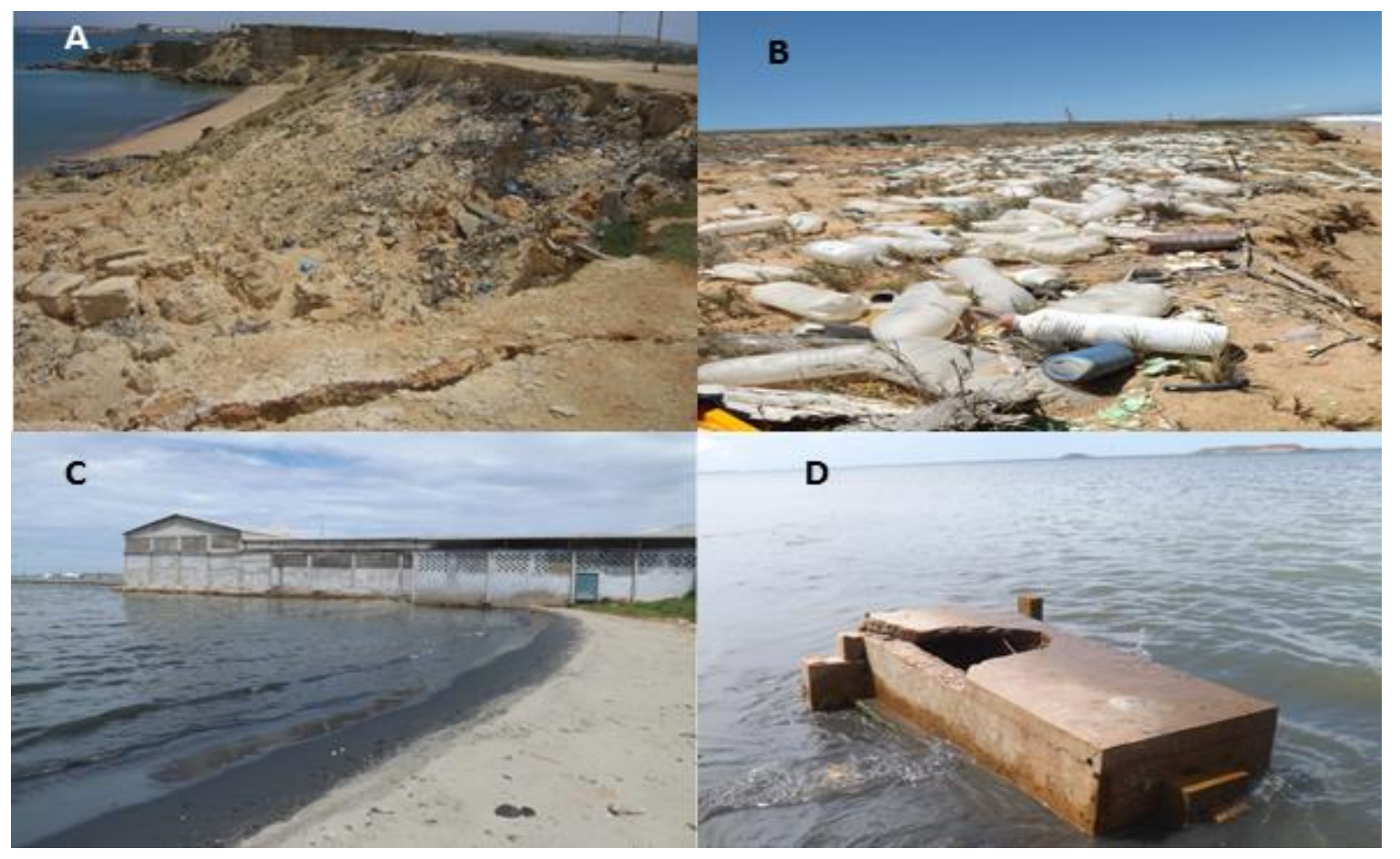

Figura 1. Contaminación ambiental. (Obsérvese en los detalles: (A) desechos sólidos en las cárcavas al sur del Castillo de Araya; (B) acumulación de botellas en Playa Brava, costa este de la Península de Chacopata; (C) Contaminación marina por aguas residuales provenientes del procesamiento de Arca zaeba (Pepitonas) en Chacopata; y (D) Féretro ubicado en el mar en la costa este de Chacopata como consecuencia del desplome del cementerio debido a la acción erosiva del oleaje sobre la base del acantilado en donde este se ubicaba). 
De allí que debemos resaltar el papel preponderante de la Geografía, como vía para la comprensión integral de los principales problemas ambientales, aunado a su rol pedagógico como orientadora para la toma de conciencia sobre las causas que lo han generan, así como, las posibles acciones mitigantes.

Es válido acotar lo referido por Gurevich et. al. (1995), quienes advierten que debemos entender que la geografía como ciencia social estudia los aspectos territoriales de los desenvolvimientos sociales, por lo que este tipo de fenómenos son parte de su objeto de estudio.

En este mismo orden de ideas, emerge como parte de las problemáticas ambientales estudiadas durante el trabajo de campo, la desigualdad social, cuyas demostraciones en la realidad, nos las refiere el Informante 9, quien expresó que:

llegamos a sitios en donde la población es pudiente, pero también vamos a lugares en donde la población es muy pobre, ejemplo cuando llegamos al morro de Barcelona, vimos que hay edificios en donde la población tienen yate, lanchas y tienen plata, pero cuando vamos a Chacopata en Araya, en dónde viven personas muy pobre, las carreteras todavía son de tierra, las casas de barro todavía, son problemas que abordamos en la discusión.

La situación referida anteriormente, permite introducir en el proceso de socialización de los saberes, temas como: pobreza, marginalidad, patrones de asentamiento y movilidad de la población, distribución de los recursos, desigualdad social y uso del espacio, que forman parte del análisis que desde la geografía se deben promover a partir de patrones de comparación como: tipo de vivienda, vías de comunicación, medios de comunicación, que mostramos en la figura 2, correspondientes a la dimensión "estándares de vida" del "Índice de Pobreza Multidimensional" (IPM) del Programa de Naciones Unidas para el Desarrollo (PNUD), a fin de generar una comprensión totalística de las realidades en estudio. 


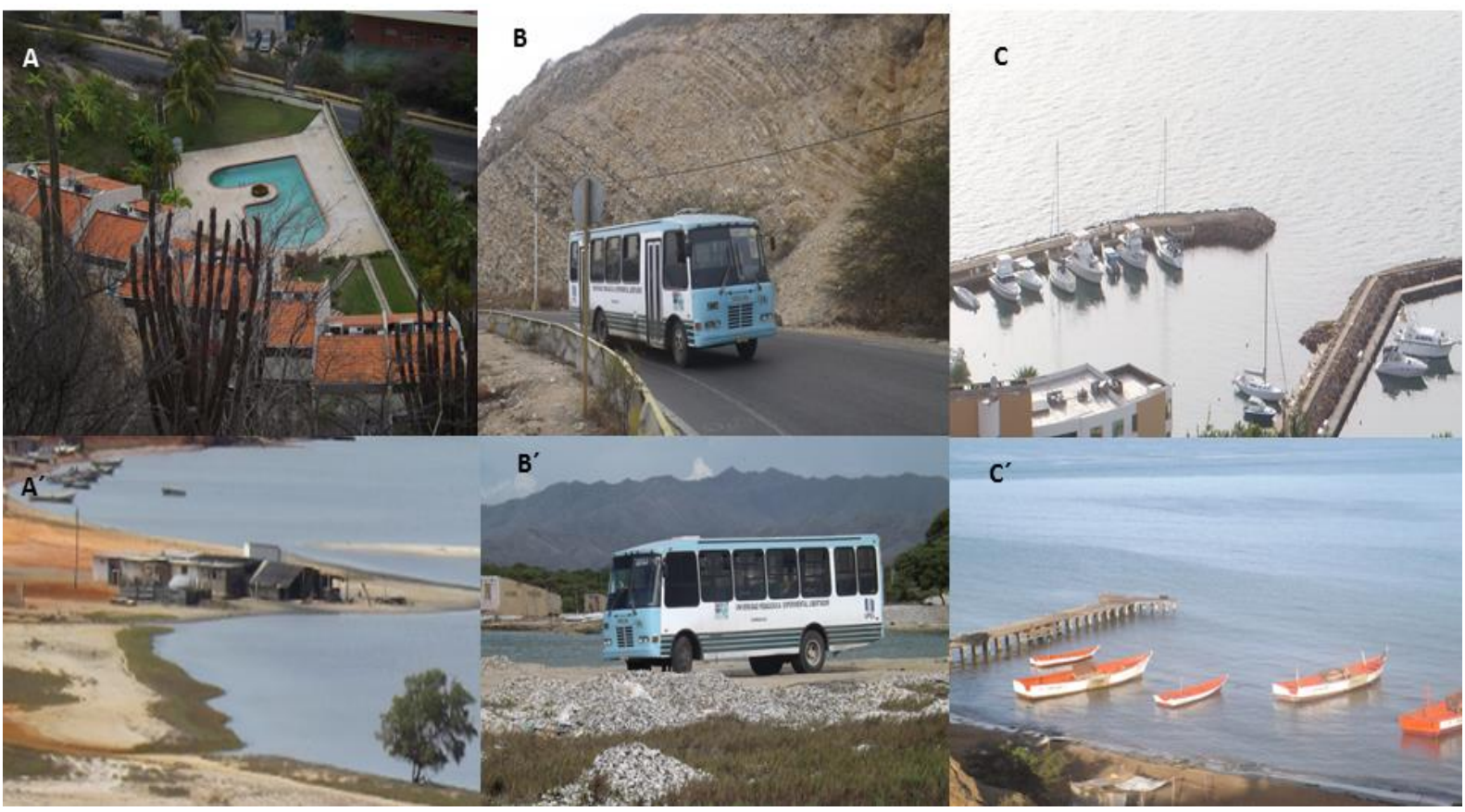

Figura 2. Desigualdad social. (La fila superior corresponde a mostraciones en el Morro de Lechería (estado Anzoátegui), mientras que la fila inferior presentamos los elementos observados en Chacopata (estado Sucre). Ambos sectores fueron referidos por los informantes. Obsérvese en los detalles: (A y A') el tipo de vivienda, (B y B') vías de comunicación, y (C y C') medios de comunicación marítimo).

\section{CONCLUSIONES}

Es común en los estudiantes del Profesorado de Geografía e Historia de la Universidad Pedagógica Experimental Libertador en su Instituto Pedagógico de Caracas, concebir al ambiente "como todo aquello que nos rodea", tal acepción, si bien es cierto que corresponde a la lógica positivista y se sustenta epistémicamente en el naturalismo y el antropocentrismo, es en esencia reduccionista, ya que por un lado, divorcia al ser humano de su relación con los otros, "lo que nos rodea", aunado a que imposibilita superar la visión recursista o utilitaria que se forja en los estudiantes, sobre el "medio ambiente".

Siendo así, interesa que los estudiantes aprendan la importancia de los aspectos físico-naturales como elementos supraordenadores de lo ambiental, y en consecuencia, humanos y naturaleza corresponden a dos entidades diferenciadas, en dónde el hombre es externo al "medio ambiente".

Ahora bien, como fue develado, el trabajo campo, propicia en los estudiantes, una noción de ambiente que supera la ya referida concepción reduccionista, para alcanzar una visión totalística e integradora, que les permita reconocer y analizar la multiplicidad de elementos que constituyen la realidad objeto de estudio.

Siendo así, con base en el testimonio de los informantes luego de su experiencia en el trabajo de campo, así como producto de su dialógica de saberes, se puede afirmar que el ambiente es la manifestación que emerge a partir de las complejas interrelaciones que se producen entre las acciones antrópicas (sociales-culturales y económicas), con el medio físico-natural, en donde el ser humano, si bien constituye sólo un elemento en esa compleja red de interrelaciones, es en esencia, el ser cognoscente, que debe procurar 
potenciar su sensibilidad y concienciación sobre los posibles impactos que sus acciones, a fin de mitigarlos de manera responsable, en procura del equilibrio planetario, $\mathrm{y}$, en definitiva de la existencia de la vida misma.

Esto supone una visión del ambiente, en dónde se comprende que la lluvia, las rocas, el mar, las aves, los reptiles, el ser humano, entre otros, se perciben como un todo, un conjunto de relaciones y conexiones interconectadas, en atención a que formamos parte de un equilibrio ecológico, constituido por un conjunto elementos bióticos y abióticos, interrelacionados por estructuras precisas y complejas, con funciones específicas para generar la vida en el planeta.

De allí que se asume que el trabajo de campo, como estrategia para la formación del docente en Geografía e Historia, propicia que el conocimiento geográfico se eleva por sobre las parcelas de la especialización, para abordar lo ambiental desde la óptica pluridimensional y compleja, que ofrece cada una de sus disciplinas, esta vez interrelacionadas en lo que llamaremos una Geografía Compleja, en dónde: la geografía física aporte las condiciones fisiográficas del espacio, la geografía humana todo lo vinculado a la dinámica poblacional, la geografía económica, lo concerniente al uso del espacio, la distribución de las riquezas y las actividades económicas, la geografía histórica lo relativo a la evolución del uso del espacio, entre otros aspectos.

Sin duda, en la aproximación vivencial a los fenómenos observado, el estudiante rompe con el paradigma antropocéntrico que supone que los seres humanos "como especie superior" tenemos derecho a usar de manera indiscriminada los espacios sin considerar a otros organismos e incluso los procesos propios de la dinámica terrestre, para vernos como un ser que tenemos responsabilidades ecológicas y que de nuestras acciones depende incluso nuestra propia existencia.
Esto, en palabras de Ferry (1992), "permite establecer la diferencia entre un amor a la vida todavía homocéntrico, reservado a los seres humanos, y un amor holista, referido al todo biogénico de que depende directa o indirectamente nuestra existencia" (p. 39).

\section{REFERENCIAS}

Aichino, G., Arancibia, L., Astegiano, N., Asis, Y., Barrera, E., Cavanagh, E., Cisterna, C., González, D., Luna, L., Palladino, L., Pedrazzani, C. y Rodigou, J. (2013). Trabajo de campo y formación del geógrafo. Algunos aportes para su reflexión. Cardinalis. [Revista en línea], 1. Disponible:

http://publicaciones.ffyh.unc.edu.ar/in dex.php/geo [Consulta: 2013, Diciembre 19]

Álvarez, L. (2007). El estudio del aprendizaje desde el modelo asociacionista y el modelo funcionalista: un recorrido histórico. Informes Psicológicos [Revista en línea], 9. Disponible:

dialnet.unirioja.es/descarga/articulo/5229 791.pdf [Consulta: 2014, Mayo 11]

Aristimuño, A. (2005). El docente como constructor de relaciones entre personas. Didac [Revista en línea], 46. Disponible: http://www.uia.mx/web/files/didac /46.pdf [Consulta: 2015, Marzo 10]

Azqueta, D. (2002). Introducción a la economía ambiental. España: McGraw Hill.

Barrera, M. (2010). Modelos epistémicos en investigación y educación. Caracas: Ediciones Quirón.

Braslavsky, C. (2002). Editorial: La demanda aumenta pero las condiciones siguen siendo insuficientes. Perspectivas [Revista en línea], 123. Disponible:

http://www.ibe.unesco.org/fileadmin/ user_upload/archive/Publications/Pro spects/ProspectsEditorials/pr123eds.p df [Consulta: 2018, Octubre 5]

Bustos, C. (2009). La problemática de los desechos sólidos. Economía [Revista en línea], 
Disponible:http://www.saber.ula.ve/bitstrea m/123456789/30305/1/articu lo5.pdf [Consulta: 2015, Julio 18]

Chew-Hung, C (2012). A Critical Discourse of Singapore's School Geography for the Twenty-First Century. Literacy Information and Computer Education Journal (LICEJ), Volume 3, Issue 3. Disponible: http://www.infonomicssociety.org/LICE

J/A\%20Critical\%20Discourse\%20of\%20 Singapore\%20School\%20Geography $\% 20$ for $\% 20$

the\%20Twenty_First\%20Century.pdf [Consulta: 2015, Febrero 12]

Ferry, L. (1992). La Ecología profunda. Revista Vuelta [Revista en línea], 192. Disponible:http://www.uv.mx/mie/files/ 2012/10/SEION4-9Sept-Ecologia-

ProfundaFerry.pdf [Consulta: 2015, Agosto 08]

Fullan, M. (2002). Las fuerzas del cambio. Explorando las profundidades de la reforma educativa. Madrid: Akal.

Godoy, I., y Sánchez, A. (2006). El trabajo de campo en la enseñanza de la Geografía. Sapiens, Revista Universitaria de Investigación [Revista en línea], 8. Disponible:

http://www2.scielo.org.ve/pdf/sp/v8n2/a rt09.pdf [Consulta: 2013, Diciembre 19]

Gutiérrez, P. (2005). El maestro del siglo XXI, Co-constructor de una sociedad humanizante. Didac [Revista en línea], 46. Disponible: http://www.uia.mx/ web/files/ didac/46.pdf[Consulta: 2015, Marzo 10]

Gurevich, R., Blanco, J., Fernández, M. y Tobío, O. (1995) Notas sobre la enseñanza de una geografía renovada. Buenos Aires: Aique Grupo Editor, S.A.

Husserl, E. (1900). Investigaciones lógicas. Madrid: Alianza.

Husserl, E. (1913). La idea de la fenomenología [Documento en línea]. Traducido por Jesús Adrián Escudero (2012) Disponible:

http://www.casadellibro.com/libro-laide a-de-la- fenomenologia/9788425428371/19

61681[Consulta: 2014, Febrero 09]

Husserl, E. (1929). Meditaciones cartesianas. México: Fondo de la cultura económica.

Husserl, E. (1970). Philosophie der Arithmetik. Mitergänzenden Texten (1890-1901), Londres: Den Haag, Martinus Nijhoff, Kluwer Academic Publisher.

Jonassen, D. (1991). Evaluating Constructivistic Learning.En T. Duffy y D. Jonassen (Edit.), Constructivism and the technology of instruction: A conversation [Libro en línea]. Lawrence Erlbaum Associates, Publishers, Estados Unidos. Disponible:

https://books.google.co.ve/books?id=4Q hgjtkobIUC\&printsec $=$ frontcover\&hl=es[ Consulta: 2015, Mayo 11]

Kanyampa Nakazwe, M. (2011). Effectiveness of Unza trained degree holder geography teachers in executing the field project in Zambian high schools of Lusaka and Kafue districts[Documentoen línea].Trabajo de grado de maestría no publicado, Universidad de Zambia. Disponible: http://dspace.unza.zm:8080/xmlui/bitst ream/

handle/123456789/867/kayampa.PDF?s equence $=1$ [Consulta: 2015, Febrero 12]

Lara, S. (2011). Las vivencias estudiantiles del trabajo de campo y sus implicaciones pedagógicas. Revista de Investigación [Revista en línea], 73. Disponible: https://www.redalyc.org/articulo.oa?id= 376140388009 [Consulta: 2017, Diciembre 19]

Llanos, E. (2010). El trabajo de campo como elemento fundamental en la enseñanza de la Geografía Histórica de las ciudades: el caso de Barranquilla. Revista del Instituto de Estudios en Educación, 13. Disponible: http://biblioteca.universia.net/ html_bura/ficha/params/title/traajo-fundam-caso.html [Consulta: 2013, Diciembre 19]

Martínez, M. (1998) La investigación cualitativa etnográfica. Manual teóricopráctico. México: Editorial Trillas.

Moliner, O. Fernández, R. y Sales, A. (2005).El profesorado de la educación básica ante 
la diversidad del alumnado. Didac [Revista en línea], 46. Disponible: http://www.uia.mx/web/files/didac/46. pdf [Consulta: 2015, Marzo 10]

Montilla, A. (2005). El trabajo de campo en la enseñanza de la Geografía. Geoenseñanza, [Revista en línea], 10. Disponible: http://www.redalyc.org/pdf/

360/36010205.pdf [Consulta: 2013, Diciembre 19]

Núñez Ravelo, F. (2018). Axiología del trabajo de campo en Geografía Física: Una teorización fenomenológica. Revista de Educación. [Revista en línea], 14. Disponible:

https://fh.mdp.edu.ar/revistas/index.php/ r_educ/article/view/2567/2919

[Consulta: 2018, Diciembre 1]

Perrenoud, P. (2007). Diez nuevas competencias para enseñar. México: Editorial Grao, Colofón

Pulgarín, R. (1998). La excursión escolar como estrategia didáctica en la enseñanza de la Geografía. Gaceta Didáctica de la Universidad de Antioquia [Revista en línea], 2. Disponible: http://www.uruguayeduca. edu.uy/P0001\% 5CFile\%5.pdf [Consulta: 2013, Diciembre 19]

Ramírez, M., Gouveia, E. y Lozada, J. (2011). El trabajo de campo estrategia metodológica para estudiar las comunidades. Revista Omnia [Revista en línea], 3. Disponible: http://www.redalyc.org/pdf/737/73720 790002.pdf [Consulta: 2013, Diciembre 19]
Rivas, S. y Morales, O. (2006). Importancia del trabajo de campo en las Ciencias Sociales. Geoenseñanzas, [Revista en línea], 2. Disponible: http://www.redalyc. org/pdf/360/36012425009.pdf [Consulta: 2013, Diciembre 19]

Romero, G. y Maskrey, A. (1993). Como entender los desastres naturales. En Maskrey (Comp.), Los desastres no son naturales [Libro en línea].Red de Estudios Sociales en Prevención de Desastres en América Latina. Disponible: http://www.oei.es/ decada/portadas/Desnat.pdf [Consulta: 2015, Julio 28]

Salas, F. (2002). Epistemología, educación y tecnología educativa. Revista de Educación. [Revista en línea], 26 (1). Disponible:

http://www.redalyc.org/pdf/440/44026 102.pdf [Consulta: 2015, Mayo 10]

Universidad Rafael Landívar (2004). Perfil ambiental de Guatemala. Informe sobre el estado del ambiente y bases para su evaluación sistemática [Documento en línea], Disponible:

http://geog.ucsb.edu/ carr/DCarr_Publicat ions/Gellertetal\%20-

\%20Dinamica\%20poblacional.pdf

[Consulta: 2015, Julio 22]

Vaillant, D. (2005). Formación de docentes en América Latina. Re-inventando el modelo tradicional. Barcelona: Octaedro

Vegas, M. (2006). Introducción a la psicología cognitiva. México: Alianza 